\title{
Near real-time coordinate estimation from double-difference GNSS data
}

Daniel Arnold $^{1} \quad$ Simon Lutz $^{1} \quad$ Rolf Dach $^{1} \quad$ Adrian Jäggi $^{1}$ Jens Steinborn ${ }^{2}$

\author{
${ }^{1}$ Astronomical Institute, University of Bern \\ ${ }^{2}$ SpaceTech GmbH, Immenstaad am Bodensee \\ IAG Scientific Assembly 2013 \\ Potsdam, Germany \\ 02.09.2013
}




\section{Near real-time GNSS data processing}

- Wide range of important applications: Hazard monitoring, atmospheric sensing, network monitoring...

- Typical approach is based on a Precise Point Positioning (PPP)

- Advantages of PPP:

- GNSS stations can be processed independently

- Efficient method to process many stations $\rightarrow$ parallelize

- Disadvantages of PPP:

- Accuracy of derived results mainly depends on the quality of used satellite clock estimates and consistency w.r.t. external network solution providing the satellite clocks

- Ambiguity resolution (AR) becomes much more involved

- Potential of classical double-difference approach for near real-time applications 


\section{National Multi-Hazard Early Warning System}

SpaceTech GmbH provides the GPS reference stations for the establishment of an early warning system in the Sultanate of Oman, in cooperation with GFZ Potsdam and AIUB.

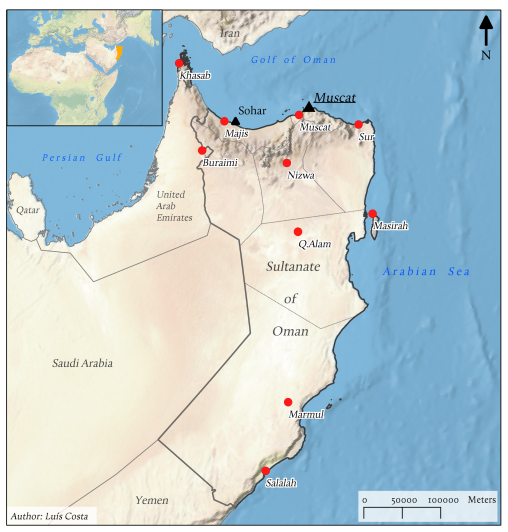

- Seismic stations, GPS stations, meteo stations, tide gauges

- GPS network: 10 permanent stations. Cross-validate possibility of a tsunami after earthquake $>5$ on Richter scale

- Requirement: Obtain displacement vectors within less than 2 minutes. 
To simulate the task of the GPS network in the early warning system, the following test environment was established:

- Warning stations: 9 European Permanent Network (EPN) stations providing real-time data, $1 \mathrm{~s}$ RINEX observation files streamed using BKG Ntrip Client (BNC).

- Reference stations: 4 IGb08 stations, 30 s hourly RINEX files dowloaded from FTP server.

- Rapid and ultra-rapid products from Center for Orbit Determination in Europe (CODE).

- Bernese GNSS Software v5.2.

- Server: 8 core Intel XEON E5-2660 2.2 GHz, 32 GB DDR3 memory. 
Geographical distribution of the stations:

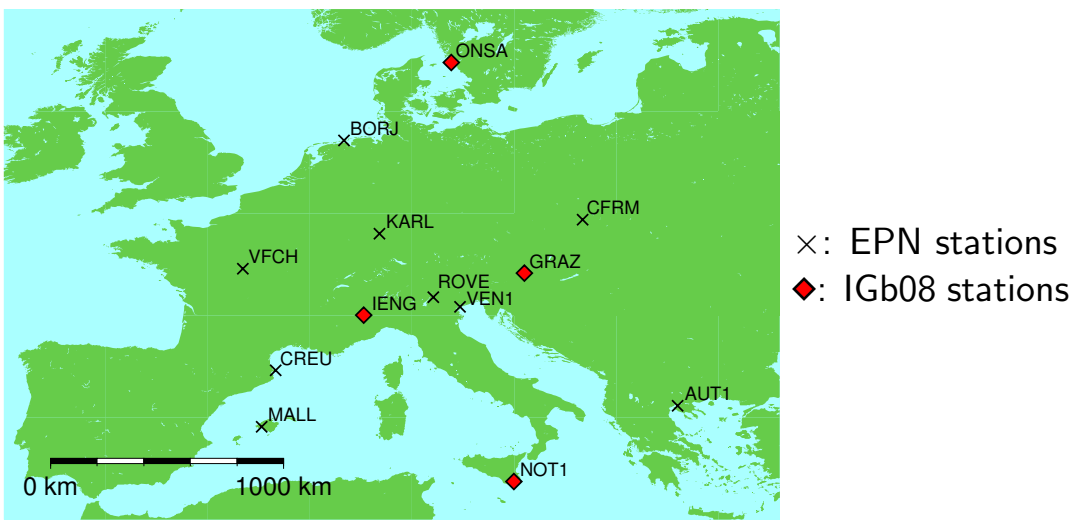




\section{Processing strategy}

The processing is divided into two main steps:

1. Datum (once per hour): Estimate static coordinates of warning and reference stations using a double-difference solution with extended ambiguity resolution (AR) procedure for different baseline lengths:

- Code- and phase-based wide-lane AR

- Code- and phase-based narrow-lane AR

- Quasi-lonosphere-Free (QIF) AR

- Direct L1/L2 AR

Use (e.g.) 8 hours of data. Yields a priori coordinates for step 2.

2. Near real-time (every 2'): Estimate kinematic coordinates of warning stations only, using a double-difference solution with QIF AR. Use (e.g.) 8 hours of data for AR and the last 3 minutes for kinematic coordinates. 


\section{Processing strategy}

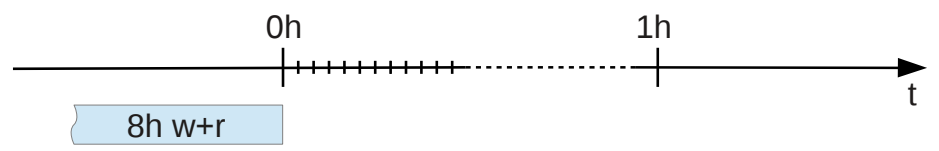

w: warning stations, $r$ : reference stations 


\section{Processing strategy}

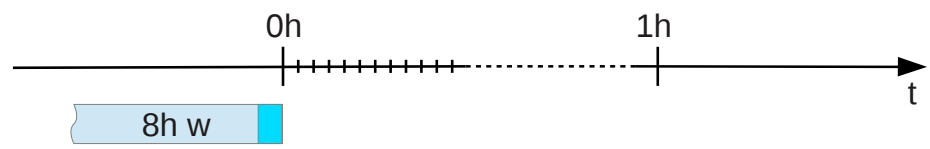

w: warning stations, $r$ : reference stations 


\section{Processing strategy}

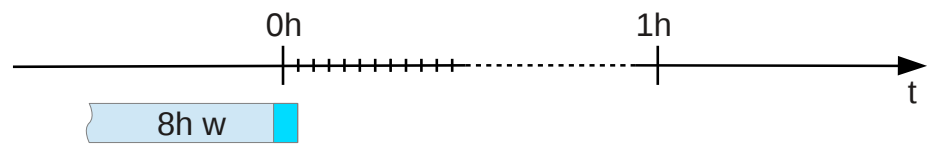

w: warning stations, $r$ : reference stations 


\section{Processing strategy}

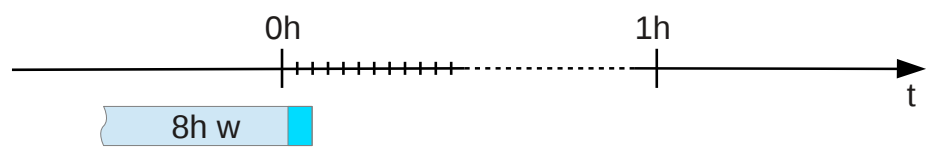

w: warning stations, $r$ : reference stations 


\section{Processing strategy}

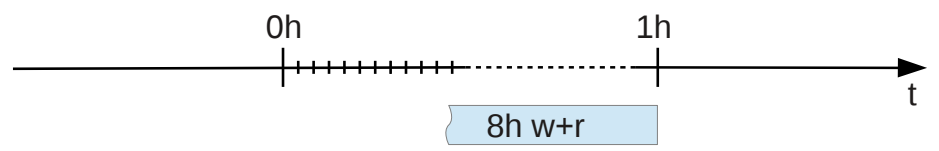

w: warning stations, $r$ : reference stations 


\section{Processing strategy}

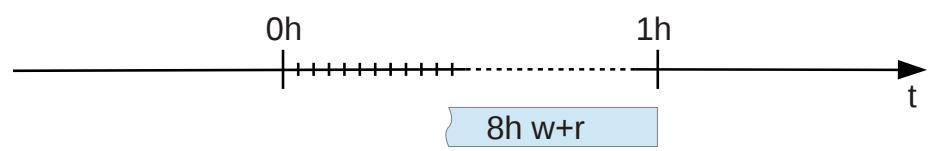

w: warning stations, $r$ : reference stations

Comments:

- Reason for split-up into 2 steps:

- Not many reference stations $\rightarrow$ long baselines $\rightarrow$ extended AR strategies (wide-lane, Melbourne-Wübbena + narrow-lane).

- Reference stations would need to provide real-time data. 


\section{Processing strategy}

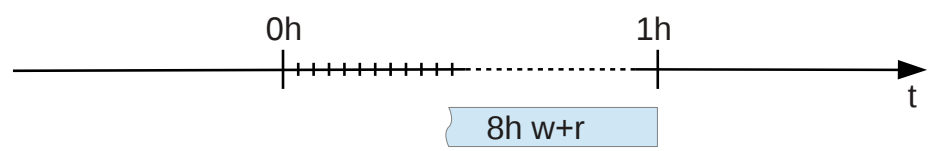

w: warning stations, $r$ : reference stations

Comments:

- Reason for split-up into 2 steps:

- Not many reference stations $\rightarrow$ long baselines $\rightarrow$ extended AR strategies (wide-lane, Melbourne-Wübbena + narrow-lane).

- Reference stations would need to provide real-time data.

- (Pre)processing is consequently parallelized: 2 inversions of the full normal equation system, otherwise station by station or baseline by baseline. 


\section{Kinematic coordinates}

Kinematic coordinates ( $3 \mathrm{~min}$ ), differences to a priori coordinates:
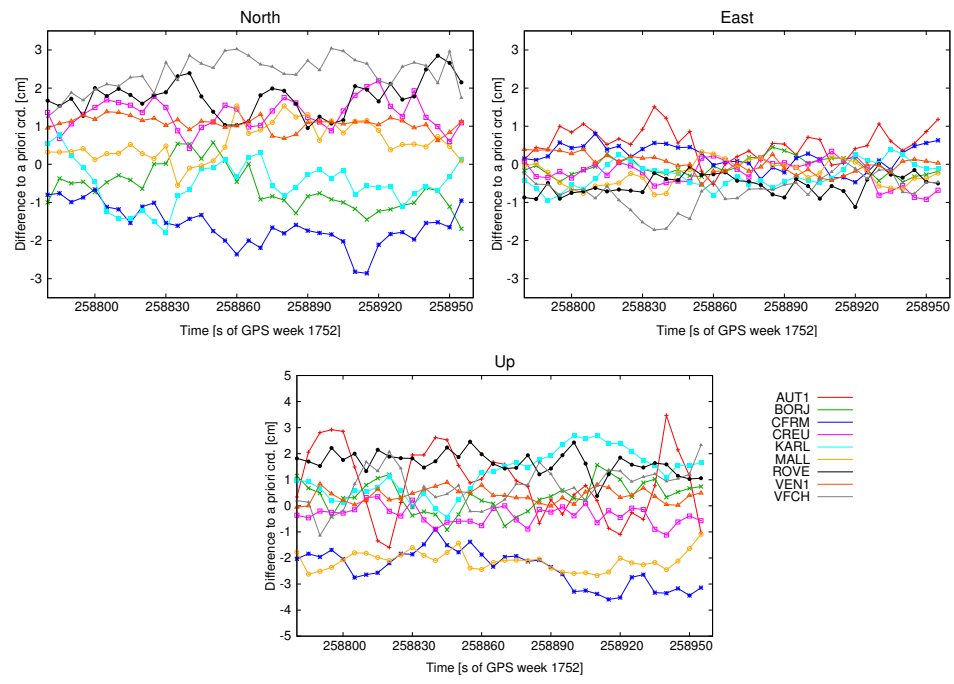


\section{Kinematic coordinates}

Overlaps of $3 \mathrm{~min}$ batches for one station (MALL):
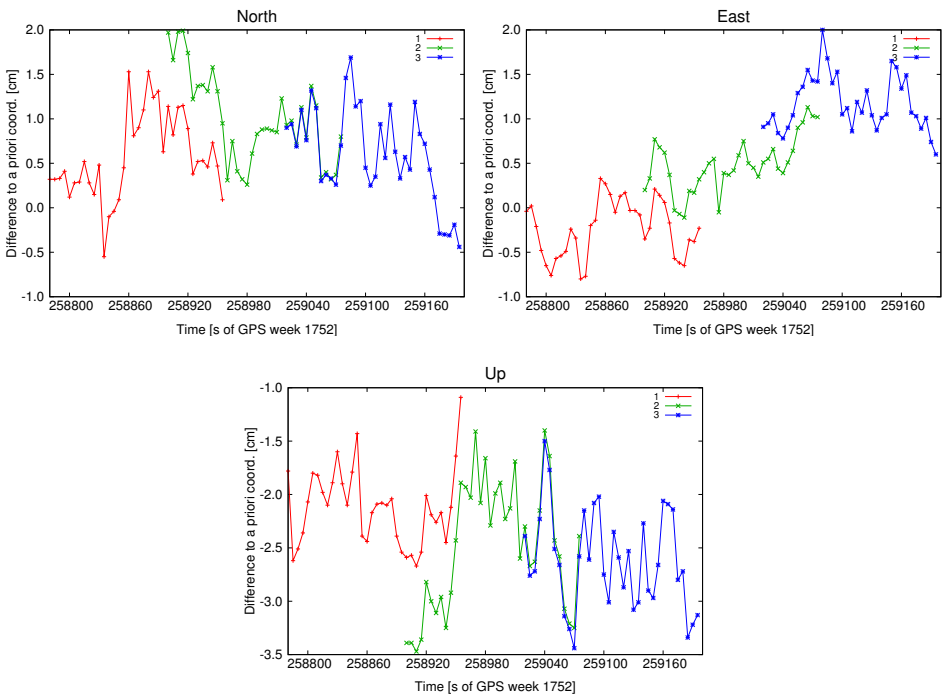


\section{How much data?}

Analyze overlaps of two consecutive 3 min batches ( 1 min overlap). Mean value and standard deviation of differences vs. total amount of data for one station (VFCH):
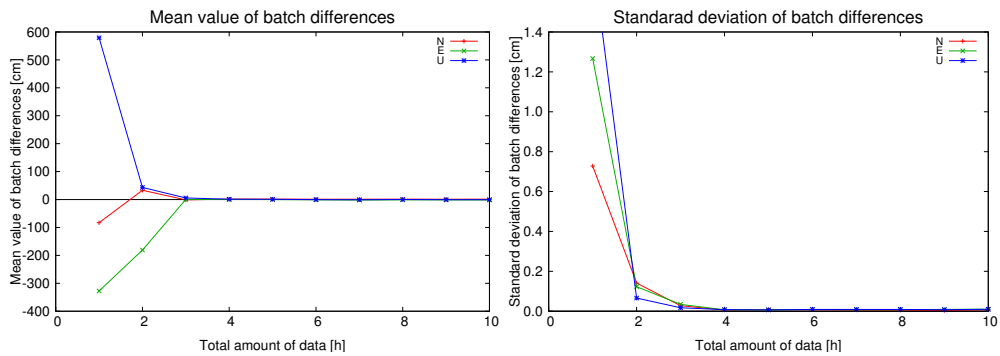


\section{How much data?}

QIF ambiguity resolution success rate vs. total amount of data:

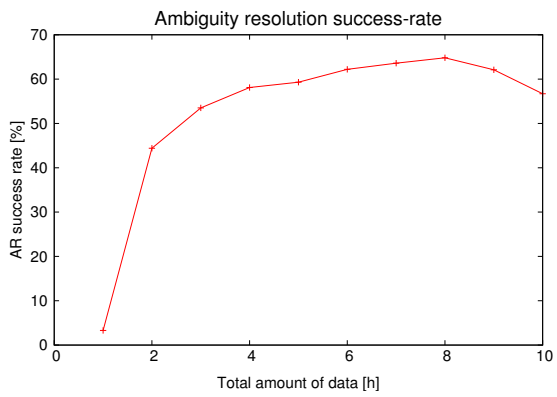

$\Rightarrow$ Enough data is needed for the processing, even if one is interested only in a few minutes of coordinates at the end! 


\section{PPP vs. double-difference}

Comparison with results $(2 \mathrm{~h})$ of a PPP (rapid products of CODE, station KARL):
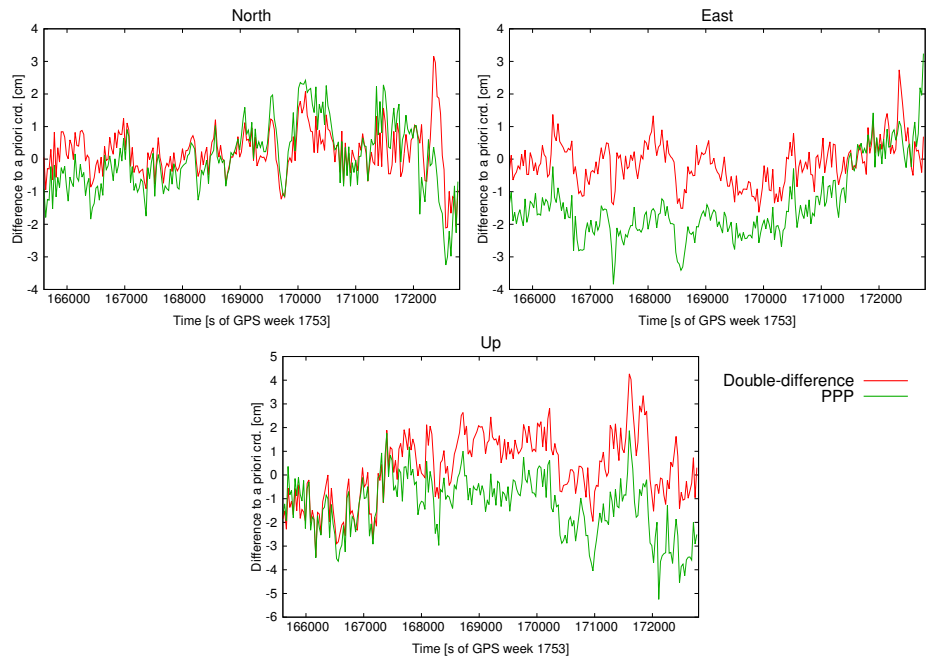

Double-difference PPP 


\section{PPP vs. double-difference}

Comparison with results $(2 \mathrm{~h})$ of a PPP (rapid products of CODE, station KARL):
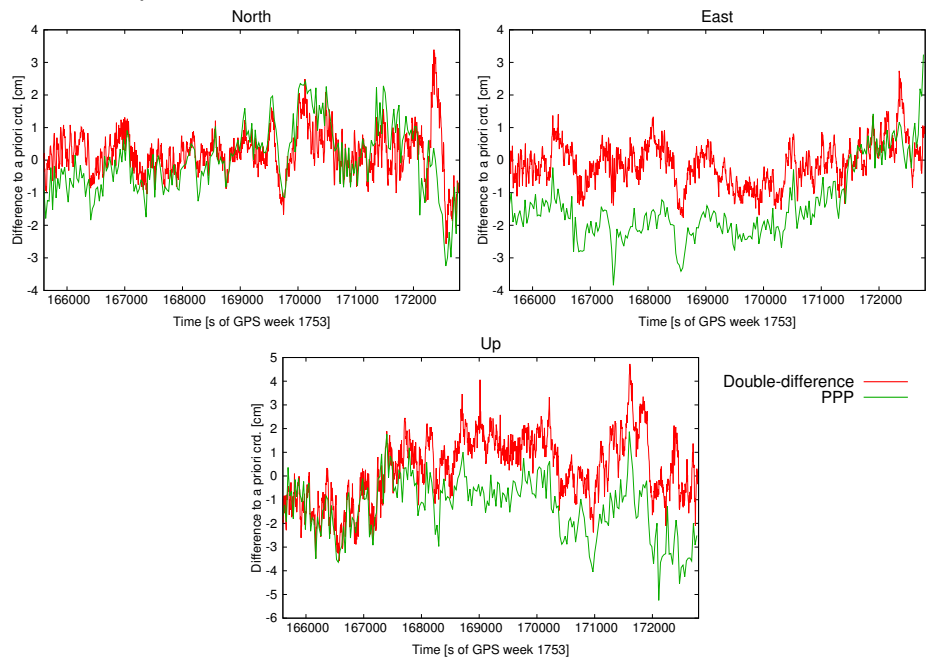

Double-difference PPP 


\section{Conclusion}

- Classical double-difference coordinate estimations can be well suited for near real-time applications.

- Independence of accurate satellite clocks. Higher sampling.

- There is a lot of potential for parallelization.

- Even if kinematic or static coordinates of only a few minutes are needed, an efficient ambiguity resolution needs enough data $(>5$ hours). 